\title{
Growth twins in high stacking fault energy metals: microstructure, texture and twinning
}

\author{
Leonardo Velasco ${ }^{\mathrm{a}}$ and Andrea M. Hodge ${ }^{\mathrm{a}, \mathrm{b},{ }^{*}}$ \\ ${ }^{a}$ Department of Chemical Engineering and Materials Science, University of Southern California, \\ Los Angeles, California 90089, USA \\ ${ }^{\mathrm{b}}$ Department of Aerospace and Mechanical Engineering, University of Southern California, Los \\ Angeles, California 90089, USA \\ *Corresponding author. Tel.: +1 213740 4225; email: ahodge@usc.edu
}

\begin{abstract}
This work explores the formation of twin boundaries in high stacking fault energy metals (SFE > $\left.125 \mathrm{~mJ} / \mathrm{m}^{2}\right)$ by synthesizing thick films $(>10 \mu \mathrm{m})$ of $\mathrm{Al}, \mathrm{Al}-5.3 \mathrm{wt} . \% \mathrm{Mg}$, and $\mathrm{Ni}$ using magnetron sputtering. We report the observation of twin boundaries that are inclined with respect to the film growth direction across the entire thickness of the films. The formation of these inclined twin boundaries results in localized changes in the texture of the columnar grains. Microstructural analysis revealed that the fraction of twinned grains in the $\mathrm{Al}$ film (46\%) is four times higher compared to other similar studies in $\mathrm{Al}$, while the Al-5.3wt.\% $\mathrm{Mg}$ and $\mathrm{Ni}$ films can be considered highly twinned since the fraction of twinned grains is $70 \%$ and $90 \%$, respectively. The experimental observations provide an explanation on the formation of twin boundaries during the synthesis of the films, and emphasize that, in addition to stacking fault energy restrictions, high grain boundary mobility is a limiting factor on the nucleation of twin boundaries.
\end{abstract}

Keywords: Nanotwins; Sputtering; High stacking fault energy metals; Twinning. 


\section{Introduction}

In crystalline solids, the propensity for twin boundary (TB) formation is highly correlated to the stacking fault energy (SFE) of the material; the lower the SFE, the higher the probability for TB formation [1]. In the case of low to intermediate $\mathrm{SFE}\left(<45 \mathrm{~mJ} / \mathrm{m}^{2}\right)$ metals such as $\mathrm{Ag}, \mathrm{Cu}$ or $\mathrm{Cu}$ alloys, films with a high density of TBs have been successfully synthesized by a variety of methods [1-8]. The inclusion of TBs in the film microstructure for low-intermediate SFE metals has shown a direct impact on the properties of the material, where the mechanical, thermal, and chemical properties are enhanced [1-8]. These improved properties have increased the working space of nanotwinned (nt) metals and contribute to the development of fundamental research for material's behavior at the nanoscale, particularly in low SFE metals with high TBs density [2, 5, 9-14].

In contrast, in metals such as $\mathrm{Ni}$, and $\mathrm{Al}$ which have a high $\mathrm{SFE}\left(125 \mathrm{~mJ} / \mathrm{m}^{2}\right.$ and $166 \mathrm{~mJ} / \mathrm{m}^{2}$, respectively $[15,16])$, TB formation is unlikely. During the last decade, research efforts have been made concerning the synthesis of TBs in $\mathrm{Al}$ and $\mathrm{Al}$ alloys at both the macroscale and at the nanoscale [17-21]. At the nanoscale, a study by Xue et al. has shown the occurrence of a few TBs in a low fraction of grains $(<9 \%)$ in thin $(<80 \mathrm{~nm}) \mathrm{Al}$ films [22]. In Ni, the synthesis of TBs has been mainly achieved by annealing, and to a lesser extent by electrodeposition and sputtering [23-25] but only the electrodeposited Ni presented TBs at the nanoscale [25]. Despite the many efforts to introduce TBs in high SFE metals, the density of TBs in high SFE materials is limited [1]. Thus the possibility of enhancing mechanical, thermal or chemical properties by introducing TBs in high SFE metals requires further development. 
In this work, thick films $(\sim 10 \mu \mathrm{m})$ of three different high SFE systems (Al, Al-5.3wt.\% Mg, and Ni) were magnetron sputtered to elucidate the formation and density of TBs in high SFE metals

$\left(\mathrm{SFE}>125 \mathrm{~mJ} / \mathrm{m}^{2}\right)$. A detailed description of the film microstructures was conducted, where it was found that the TB plane is usually inclined with respect to the film growth direction and it is not perpendicular to the [lll 111$]$ grain growth direction. These results are contrary to the observations in magnetron sputtered nt metals with low SFE, where the TBs are perpendicular to the film growth direction [4, 26-30]. Further analysis of the microstructure revealed changes to the texture of the columnar grains due to the inclined TBs. A comparison between the nanostructural features of the three high SFE metals revealed that the three films have higher TB density and fraction of twinned grains than previous values reported in literature [22, 24]. The physical evidence shows that in addition to SFE restrictions, the formation and stability of nucleated TBs can be limited by high grain boundary mobility during the synthesis of the films. Overall, the experimental observations allowed discussion on the formation of TBs in high SFE metals and provide an explanation regarding the nucleation of TBs during continuous grain growth in magnetron sputtering.

\section{Experimental details}

$\mathrm{Ni}$ (99.995\% purity), Al (99.99\% purity), and Al-5.3wt.\% Mg (99.99\% purity) targets (7.62 cm in diameter) were magnetron sputtered onto $2.54 \mathrm{~cm} \mathrm{Si} \mathrm{(100)} \mathrm{substrates,} \mathrm{the} \mathrm{SFEs} \mathrm{of} \mathrm{these} \mathrm{three}$ materials is within the range of $125 \mathrm{~mJ} / \mathrm{m}^{2}-166 \mathrm{~mJ} / \mathrm{m}^{2}[15,16]$. Although, there is no specific value for the SFE of the Al-5.3wt.\% Mg, it can be assumed that it is slightly less than the SFE of pure $\mathrm{Al}\left(166 \mathrm{~mJ} / \mathrm{m}^{2}\right)$ based on computational studies by Muzyk et al. [31] and experiments by Kannan et al. in Al-1\% Mg [32]. For simplicity, the Al-5.3wt.\% Mg alloy film will be referred to as Al-Mg. The sputtering chamber was evacuated prior to deposition to a base pressure $<1.2 \mathrm{x}$ 
$10^{-6}$ Torr $(1$ Torr $=133.3 \mathrm{~Pa})$. During sputtering, the Ar working pressure was 2 mTorr, the target-substrate distance $7.62 \mathrm{~cm}$, and the deposition rate $>7 \mathrm{~nm} / \mathrm{sec}$. The thickness of the synthesized films was $>10 \mu \mathrm{m}$, as measured with an XP-2 profilometer (AMBios).

The microstructural characterization of the synthesized films was conducted by transmission electron microscopy (TEM) using a JEOL JEM-2100F operated at $200 \mathrm{kV}$. The specimens for TEM analysis were prepared by two methods, mounting a cross section of the film in silicon, dimple grinding, and ion milling using a Fischione Model 1050 TEM Mill and by performing focused ion beam (FIB) lift-out using a JIB-4500 FIB (JEOL). The texture of the films was then analyzed by taking X-ray diffraction (XRD) patterns using a Rigaku Ultima IV, and by transmission electron backscatter diffraction (T-EBSD). The FIB lift-out specimens used for TEM were also used for T-EBSD, which was carried out on the cross-section of the films by using a JSM-7001F-LV scanning electron microscope (JEOL) with a Hikari detector (EDAX). The FIB lift-out specimens were tilted $20^{\circ}$ from the horizontal plane to give rise to a strong signal (more information in regards to the geometry of the experiment can be found elsewhere [33]); the accelerating voltage and working distance were $30 \mathrm{kV}$ and $15 \mathrm{~mm}$, respectively, while the step size was 5-10 $\mathrm{nm}$. The T-EBSD collated data was analyzed with the TSL orientation image microscopy (OIM) software. Data with confidence index lower than 0.1 were removed and showed as black pixels in the orientation maps. $\Sigma 3$ TBs were identified by applying boundary trace analysis as described elsewhere $[34,35]$.

\section{Results and discussion}

\subsection{Microstructural characterization}


Cross-sectional TEM images were taken to study the microstructure of the films and identify TBs. In Figures 1a, 1c, and 1e, low magnification bright field images of columnar grains with inclined TBs (marked by red dotted lines) are presented for $\mathrm{Al}, \mathrm{Al}-\mathrm{Mg}$, and $\mathrm{Ni}$, respectively. The insets depict the selected area electron diffraction (SAED) patterns obtained from the columnar grains. The typical double hexagon pattern of the (110) zone axis oriented twinned grain is highlighted by the dotted blue and magenta lines; each SAED pattern was also indexed with the corresponding plane orientation. Figures $1 \mathrm{~b}, 1 \mathrm{~d}$, and 1f show zoomed in high resolution TEM images of the square regions in Figures 1a, 1c, and 1e. The TBs are marked by the red dotted lines and lie in a $\left\{\begin{array}{llll}1 & 1 & 1\end{array}\right\}$ family plane that is not perpendicular to the [lll 11 1 $]$ direction (for

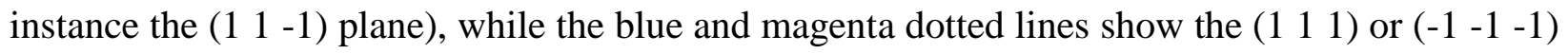
planes at each of the two sides of the TBs. The yellow lines mark the (1 $11-1)$ planes that are parallel to the (1 1 -1) TB plane. The insets in Figures $1 \mathrm{~b}, 1 \mathrm{~d}$, and 1f show the fast Fourier transform of the high resolution TEM images confirming the presence of a TB. Since the planes at each side of the TB are ( $\left.\begin{array}{lll}1 & 1 & -1\end{array}\right)$ planes (yellow lines in the high resolution images), the TBs depicted in Figures 1 are symmetrical, tilt, and twist boundaries, which is the definition used to classify $\Sigma 3\left\{\begin{array}{lll}1 & 1 & 1\end{array}\right\}$ coherent twin boundaries (CTBs) [36, 37].

Representative cross-sectional low magnification TEM images of the Al, Al-Mg, and Ni films microstructures are displayed in Figure 2 (a-c) respectively. The three microstructures have columnar grains, and several TBs are highlighted by the dotted red lines. The TBs were identified following the characterization procedures shown in Figure 1. In general, the TBs are not perpendicular to the film growth direction, which is in contrast from what is commonly observed in the synthesis of TBs in low SFE materials by magnetron sputtering [26-28, 38]. In Figure 2, $\alpha$ is the angle between the inclined TB and a plane that is perpendicular to the film 
growth direction. In Figures $2 \mathrm{a}$ and $2 \mathrm{~b}$ the TBs are preferentially inclined at an angle $\alpha \sim 70^{\circ}$, while in Figure $2 \mathrm{c}$ the TBs are inclined at different angles and $\alpha$ varies from $0^{\circ}$ to $\sim 75^{\circ}$. Notice that in Figure 1, the angle $\alpha$ between the TB plane and a plane perpendicular to the film growth direction is $\sim 70.5^{\circ}$ for both $\mathrm{Al}$ and $\mathrm{Al}-\mathrm{Mg}$ (Figures $1 \mathrm{~b}$ and $1 \mathrm{~d}$ ), while in the case of Ni (Figure 1f) $\alpha$ is $\sim 50^{\circ}$. Overall, the microstructural characterization reveals that the inclined TBs are present across the thickness of the films (Figure 2), and that the inclined TBs can induce changes in the columnar grains texture (Figure 1), which is discussed in the following section.

\subsubsection{Texture Analysis}

The experimentally observed inclined CTBs can be associated with a change in the texture of the films. For example, in the case of $\mathrm{Al}$ and $\mathrm{Al}-\mathrm{Mg}$ films at one side of the CTB, there are $\left(\begin{array}{lll}1 & 1 & 1\end{array}\right)$ planes that are perpendicular to the [lllll 111 grain growth direction as well as the film growth direction, while on the other side of the CTB, the $\left(\begin{array}{lll}1 & 1 & 1\end{array}\right)$ planes are inclined with respect to the film growth direction (Figure 1b and Figure 1d). In the case of the Ni film, the (llll 11 ) planes are inclined with respect to the film growth direction at each of the two sides of the CTB (Figure 1f). XRD and T-EBSD were used to investigate the overall film texture and to support the observations by TEM. Specifically, T-EBSD was used to identify the texture of the grains at each side of the $\Sigma 3$ TBs. Figure 3 shows the XRD patterns of the Al, Al-Mg, and Ni films. The $\mathrm{Al}$ and $\mathrm{Al}-\mathrm{Mg}$ films XRD patterns both show a strong $\{111\}$ texture, while the Ni film XRD pattern presents two strong textures $\{111\}$ and $\{200\}$.

In EBSD, a boundary is considered a $\Sigma 3 \mathrm{~TB}$ if the misorientation angle between two grains is $\sim 60^{\circ}$ and if the misorientation axis between the same two grains is perpendicular to a $\{111\}$ plane [35]. Figure 4 shows T-EBSD scans of single columnar grains from the films cross-section, 
where the colors correspond to the orientation of the grains in the film growth direction. In Figure 4 (a-d), the inverse pole figures of a columnar grain with $\Sigma 3$ TBs are identified by the change in color in each columnar grain. The legend of the inverse pole figure is included to identify the change in texture for each of the columnar grains after an inclined TB. Notice that in the case of Ni, two scans are included to show TBs that are nearly perpendicular (Figure 4c) and inclined (Figure 4d) to the film growth direction. Table 1 lists each of the columnar grains shown in Figure 4, where the planes at each side of the TBs are labeled by the white numbered circles and are perpendicular to the paper plane in Figure 4. The OIM software analysis was used to find the plane orientation for each of the white numbered circles with respect to the film growth direction, as well as the misorientation angle and misorientation axis between consecutive numbered white circles. For example, in Figure 4a the columnar grain contains three numbered circles that are also shown on the right-hand side texture legend at their corresponding orientations, while in Table 1 the plane that corresponds to the number 1 and number 2 white circles are listed as (-1 11 1 $)$ and $\left(\begin{array}{lll}-1 & 1 & 5\end{array}\right)$ respectively. The misorientation angle and the plane perpendicular to the misorientation axis between the two planes is $60^{\circ}$ and $(1-11)$, respectively. In general, the $\mathrm{Al}$ and $\mathrm{Al}-\mathrm{Mg}$ columnar grains showed mainly two textures before and after the $\Sigma 3$ TBs $\left\{\begin{array}{lll}1 & 1 & 1\end{array}\right\}$ and $\left\{\begin{array}{lll}1 & 1 & 5\end{array}\right\}$, while Ni columnar grains showed various textures, similar to the observations in the XRD patterns in Figure 3.

Despite the strong $\left\{\begin{array}{lll}1 & 1 & 1\end{array}\right\}$ texture in $\mathrm{Al}$ and $\mathrm{Al}-\mathrm{Mg}$ observed in Figure 3, the TBs were not observed in planes perpendicular to the film growth direction, or to the [ [ $\left.\begin{array}{lll}1 & 1 & 1\end{array}\right]$ grain growth direction. In the case of the Ni film, some TBs are nearly perpendicular to the growth direction of the film, but due to the fact that the grains have different orientations, it was found that mostly all the TBs are lying in a $\left\{\begin{array}{lll}1 & 1 & 1\end{array}\right\}$ family plane that is not perpendicular to the $\left[\begin{array}{lll}1 & 1 & 1\end{array}\right]$ grain 
growth direction. These results are in contrast to the observation of TBs in low SFE materials which have a strong $\left\{\begin{array}{lll}1 & 1 & 1\end{array}\right\}$ texture $[26-29,39]$. Therefore, the inclined TBs shown in this manuscript seemed to promote changes in the texture of the columnar grains.

\subsection{Microstructural features}

After evaluating the effects of TBs in the texture of the grains, it is imperative to quantify the nanostructural features of the films. This evaluation was conducted by examining several TEM images such as the ones in Figures 1 and 2. The data is presented in Table 2, which lists the SFE of the material, the TB density (amount of TBs per unit area), the total area analyzed to count TBs, the fraction of twinned grains, and the average grain width of the columnar grains for the $\mathrm{Al}, \mathrm{Al}-\mathrm{Mg}$, and Ni films (see Figure $\mathrm{S} 1$ for TEM images comparing the difference in grain width among the three films).

The TB density (TBD) was calculated by counting at least 500 TBs in each sample, while the fraction of twinned grains (FTG) and the average grain width were calculated by counting and measuring at least 125 grains in each sample. To estimate the FTG, a grain is considered to be a twinned grain if it contains at least three TBs. The Ni sample, which has the lowest SFE, presented the highest TBD (69 TBs/ $\left./ \mathrm{m}^{2}\right)$ and the highest FTG (90\%) among the three films, followed by the Al-Mg sample. The TBD in Ni is higher than in Al-Mg and $\mathrm{Al}$ by a factor of 1.5 and 20, respectively. The FTG in Ni is higher than in Al-Mg and Al by a factor of 1.2 and 2 .

The results presented in Table 2 suggest that the SFE could be the main factor that highly influences the TBD and the FTG, the lower the SFE in a material the higher the probability of TB formation [1]. However, since the SFE of the Al-Mg sample is slightly lower than that of the

Al sample (SFE $166 \mathrm{~mJ} / \mathrm{m}^{2}$ ), the TBD and the FTG in these two samples should be similar. 
Instead, the Al-Mg sample presented TBD and FTG similar to the ones observed in the Ni sample (SFE $125 \mathrm{~mJ} / \mathrm{m}^{2}$ ). The comparison in grain width among the three samples indicates that there should be another mechanism which can also influence the formation of TBs, specifically, during the film synthesis. It is possible to associate the significant difference in grain width between $\mathrm{Al}$ and the $\mathrm{Al}-\mathrm{Mg}$ or $\mathrm{Ni}$ to the formation of TBs. In this case, high grain boundary mobility (lateral grain growth) during the film synthesis can affect the nucleation and stability of TBs, which will be explained in the following section.

\subsubsection{Nucleation and stability of TBs during sputtering}

Recently, an analytical model proposed by Xue et al. suggests that the nucleation of inclined TBs is more favorable than parallel TBs in polycrystalline $\mathrm{Al}$, and that the formation of inclined TBs is less probable as the thickness of the film becomes larger than 80nm [22]. The inclined CTBs presented in this manuscript are observed across the entire thickness of the films $(\sim 10 \mu \mathrm{m})$, and the average measured length of the inclined CTBs for the three different samples is $>80 \mathrm{~nm}$ $(\mathrm{Al}=400 \pm 50 \mathrm{~nm}, \mathrm{Al}-\mathrm{Mg}=140 \pm 20 \mathrm{~nm}$, and $\mathrm{Ni}=80 \pm 9 \mathrm{~nm})$. The present results provide evidence that the nucleation of inclined CTBs for the three films is not limited to the earlier stages of film growth (thin films thickness $<80 \mathrm{~nm}$ ), which is contrary to the observations by Xue et al. [22]. Moreover, the FTG in the Al film is at least four times higher compared to the study by Xue et $a l$. , where the fraction of twinned grains is $9 \%$ [22]. Therefore, the formation of inclined CTBs must be energetically favorable and can be independent of the films thickness. Thus, the nucleation of inclined CTBs occurs in two zones: (i) during the early stages of film growth, which follows the analytical model by Xue et al.; and (ii) during the continuous grain growth of the film, which will be addressed in the next paragraph. 
In sputtering, the continuous growth of a columnar grain occurs by the arrival of atoms to the surface of the grain, where a nucleus free of defects can coalesce, forming monolayers that follow epitaxial growth $[40,41]$. The formation of a nucleus with any type of defect requires higher energy than that of a nucleus without defects. Since the SFE of the materials used in this study is high, the formation of a nucleus with a stacking fault or TB defect is expected to be unlikely. It was observed that the inclined CTBs studied in this manuscript cross the entire width of the columnar grains and that they lie in a plane that is not perpendicular to the [1 111$]$ grain growth direction. Therefore, it is proposed that during continuous growth of the film, the formation of a nucleus with an inclined CTB (similar to the one described at the early stages of sputtering by Xue et al.) can occur at the lateral edges of the columnar grain (grain boundaries are regions of high energy, where the nucleation of precipitates and second phases is feasible). In this case the area from a small portion of the columnar grain boundary can substitute the area of the ITB that is in contact with the matrix/TB plane similar to the mechanism described by Xue $e t$ al. [22]. Since the ratio between the area occupied by the columnar grain boundary and the area occupied by the inclined CTB plane can be small, it is possible that a nucleus with an inclined CTB can develop at the lateral edge of a columnar grain. Moreover, the formation of such inclined CTBs is at least 2 times more feasible than the one for a perpendicular TB (see supplementary material for a further description). If the nucleus is stable, the CTB will propagate through the entire width of the grain due to the constant arrival of atoms, and it will be terminated upon arrival to the other side of the columnar grain. The formation of the inclined CTB must be accompanied by a change in the grain growth direction, which is supported by the texture analysis conducted by T-EBSD. 
The previous explanation allows for an understanding of the substantial difference observed in the TBD and the FTG in Al compared to the other two films (see Table 2, Al-Mg and Ni). The grain width of $\mathrm{Al}$ is three times wider than that of $\mathrm{Al}-\mathrm{Mg}$ or $\mathrm{Ni}$, which can be attributed to two main factors: (i) the effect of the Zener drag force, where impurities or alloying elements can impinge grain boundaries and prevent grain growth [42] (Al vs. Al-Mg); and (ii) the difference in the melting point temperature between $\mathrm{Al}$ and $\mathrm{Ni}$, indicating that grain boundary mobility in $\mathrm{Ni}$ is lower than in Al. In any of the two cases the mobility of boundaries for $\mathrm{Al}$ will be higher than in Al-Mg or Ni. It is possible that an inclined CTB nucleus that is being formed at the columnar grain boundary is detached from the grain boundary as a result of the flux of vacancies and local distortions of the atomic arrangement (grain boundary mobility). Therefore, an inclined CTB nucleus will not be in contact with the columnar grain boundary as a result of grain boundary mobility, which makes the inclined CTB nucleus unstable, and prevents development. Hence, the observed large grain width in the $\mathrm{Al}$ film is an indicator of high grain boundary mobility during continuous grain growth. This effect could lower the formation of inclined CTBs during the Al film synthesis and explain that, in addition to the high SFE of Al, grain boundary mobility during continuous film growth affects the formation of CTBs. Therefore, by inhibiting lateral grain growth in high SFE metals, it is possible to enhance CTB nucleation, similar to the observations in the $\mathrm{Al}-\mathrm{Mg}$ or Ni film, which presented smaller grain width compare to $\mathrm{Al}$.

\section{Conclusions}

In this study, thick films $(\sim 10 \mu \mathrm{m})$ of $\mathrm{Al}, \mathrm{Al}-\mathrm{Mg}$ and $\mathrm{Ni}$ with $\mathrm{CTBs}$ were successfully synthesized by magnetron sputtering. The FTG in the $\mathrm{Al}$ film (46\%) is at least four times higher compared to other studies in $\mathrm{Al}$ by sputtering, while the $\mathrm{Al}-\mathrm{Mg}$ and $\mathrm{Ni}$ films can be considered highly twinned since they have a FTG of $70 \%$ and $90 \%$, respectively. The microstructure 
analysis revealed that the TBs lie in a $\left\{\begin{array}{lll}1 & 1 & 1\end{array}\right\}$ family plane that is not perpendicular to the [ $\left.\begin{array}{llll}1 & 1 & 1\end{array}\right]$ grain growth direction, thus the TBs induced changes in the texture of the columnar grains. The experimental observations provide an explanation to the formation of TBs during continuous grain growth of the films, and emphasizes the influence of grain boundary mobility on the nucleation of TBs in high SFE metals. Therefore, in addition to SFE restrictions, the formation of TBs during film deposition can be limited due to high grain boundary mobility, such as, in the case of the $\mathrm{Al}$ film. The overall results show that the nucleation of inclined TBs at the edges of columnar grains is at least 2 times more feasible than that of for perpendicular nucleation, and that it is possible to scale the synthesis of TBs in high SFE metals from thin films to thick films to expand the working space of metals with high SFEs.

\section{Acknowledgements}

This study was sponsored by the Defense Advanced Research Projects Agency (Grant No. N66001-12-1-4243), the National Science Foundation (Grant No. DMR-0955338) and the Office of Naval Research (Grant No. N00014-15-1-2486). The authors would like to thank Nathan Heckman at USC for his collaboration with T-EBSD analysis, as well as the Center for Electron Microscopy and Microanalysis (CEMMA) at USC for the use of their facilities.

\section{References}

[1] I.J. Beyerlein, X. Zhang, A. Misra. Growth Twins and Deformation Twins in Metals, Annual Review of Materials Research 44 (2014) 329-363.

[2] A. Stukowski, K. Albe, D. Farkas. Nanotwinned fcc metals: Strengthening versus softening mechanisms, Physical Review B 82 (2010) 224103.

[3] Y. Wei. Scaling of maximum strength with grain size in nanotwinned fcc metals, Physical Review B 83 (2011) 132104.

[4] A.M. Hodge, Y.M. Wang, T.W. Barbee Jr. Mechanical deformation of high-purity sputterdeposited nano-twinned copper, Scripta Materialia 59 (2008) 163-166.

[5] Y. Zhao, T.A. Furnish, M.E. Kassner, A.M. Hodge. Thermal stability of highly nanotwinned copper: The role of grain boundaries and texture, Journal of Materials Research 27 (2012) 3049-3057. 
[6] T.A. Furnish, A.M. Hodge. On the mechanical performance and deformation of nanotwinned Ag, APL Materials 2 (2014) -.

[7] Y. Zhao, I.C. Cheng, M.E. Kassner, A.M. Hodge. The effect of nanotwins on the corrosion behavior of copper, Acta Materialia 67 (2014) 181-188.

[8] N.M. Heckman, L. Velasco, A.M. Hodge. Influence of Twin Thickness and Grain Size on the Tensile Behavior of Fully Nanotwinned CuAl Alloys Advanced Engineering Materials (2015) n/a-n/a.

[9] O. Anderoglu, A. Misra, H. Wang, F. Ronning, M.F. Hundley, X. Zhang. Epitaxial nanotwinned Cu films with high strength and high conductivity, Applied Physics Letters 93 (2008) 083108.

[10] L. Lu, X. Chen, X. Huang, K. Lu. Revealing the Maximum Strength in Nanotwinned Copper, Science 323 (2009) 607-610.

[11] J. Wang, N. Li, O. Anderoglu, X. Zhang, A. Misra, J.Y. Huang, J.P. Hirth. Detwinning mechanisms for growth twins in face-centered cubic metals, Acta Materialia 58 (2010) 2262-2270.

[12] A.M. Hodge, T.A. Furnish, A.A. Navid, T.W. Barbee Jr. Shear band formation and ductility in nanotwinned Cu, Scripta Materialia 65 (2011) 1006-1009.

[13] X. Zhang, A. Misra. Superior thermal stability of coherent twin boundaries in nanotwinned metals, Scripta Materialia 66 (2012) 860-865.

[14] T.A. Furnish, J. Lohmiller, P.A. Gruber, J. T. W. Barbee, A.M. Hodge. Temperature-dependent strain localization and texture evolution of highly nanotwinned Cu, Applied Physics Letters 103 (2013) 011904.

[15] M. Rester, C. Motz, R. Pippan. Stacking fault energy and indentation size effect: Do they interact?, Scripta Materialia 58 (2008) 187-190.

[16] T.C. Schulthess, P.E.A. Turchi, A. Gonis, T.G. Nieh. Systematic study of stacking fault energies of random Al-based alloys, Acta Materialia 46 (1998) 2215-2221.

[17] D. Bufford, Y. Liu, Y. Zhu, Z. Bi, Q.X. Jia, H. Wang, X. Zhang. Formation Mechanisms of Highdensity Growth Twins in Aluminum with High Stacking-Fault Energy, Materials Research Letters 1 (2013) 51-60.

[18] Z. Xu, N. Li, H. Jiang, L. Liu. Deformation nanotwins in coarse-grained aluminum alloy at ambient temperature and low strain rate, Materials Science and Engineering: A 621 (2015) 272-276.

[19] F. Zhao, L. Wang, D. Fan, B.X. Bie, X.M. Zhou, T. Suo, Y.L. Li, M.W. Chen, C.L. Liu, M.L. Qi, M.H. Zhu, S.N. Luo. Macrodeformation Twins in Single-Crystal Aluminum, Physical Review Letters 116 (2016) 075501.

[20] M.W. Chen, J.W. McCauley, D.P. Dandekar, N.K. Bourne. Dynamic plasticity and failure of highpurity alumina under shock loading, Nat Mater 5 (2006) 614-618.

[21] D. Bhattacharyya, X.-Y. Liu, A. Genc, H.L. Fraser, R.G. Hoagland, A. Misra. Heterotwin formation during growth of nanolayered Al-TiN composites, Applied Physics Letters 96 (2010).

[22] S. Xue, Z. Fan, Y. Chen, J. Li, H. Wang, X. Zhang. The formation mechanisms of growth twins in polycrystalline Al with high stacking fault energy, Acta Materialia 101 (2015) 62-70.

[23] B. Lin, Y. Jin, C.M. Hefferan, S.F. Li, J. Lind, R.M. Suter, M. Bernacki, N. Bozzolo, A.D. Rollett, G.S. Rohrer. Observation of annealing twin nucleation at triple lines in nickel during grain growth, Acta Materialia 99 (2015) 63-68.

[24] R. Wang, S.D. Dahlgren. Observation of twin faults in sputter-deposited high-purity nickel, J Mater Sci 10 1456-1458.

[25] H. Shan, Y. Zhang, S. Mao, Z. Zhang. Electron microscopy study of five-fold twins in electrodeposited nano-twin Ni, Acta Metall 50 (2014) 305-312.

[26] A.M. Hodge, Y.M. Wang, T.W. Barbee Jr. Large-scale production of nano-twinned, ultrafinegrained copper, Materials Science and Engineering: A 429 (2006) 272-276.

[27] L. Velasco, M.N. Polyakov, A.M. Hodge. Influence of stacking fault energy on twin spacing of $\mathrm{Cu}$ and Cu-Al alloys, Scripta Materialia 83 (2014) 33-36. 
[28] L. Velasco, A.M. Hodge. The mobility of growth twins synthesized by sputtering: Tailoring the twin thickness, Acta Materialia 109 (2016) 142-150.

[29] X. Zhang, A. Misra, H. Wang, T.D. Shen, M. Nastasi, T.E. Mitchell, J.P. Hirth, R.G. Hoagland, J.D. Embury. Enhanced hardening in $\mathrm{Cu} / 330$ stainless steel multilayers by nanoscale twinning, Acta Materialia 52 (2004) 995-1002.

[30] X. Zhang, O. Anderoglu, R. Hoagland, A. Misra. Nanoscale growth twins in sputtered metal films, JOM Journal of the Minerals, Metals and Materials Society 60 (2008) 75-78.

[31] M. Muzyk, Z. Pakiela, K.J. Kurzydlowski. Ab initio calculations of the generalized stacking fault energy in aluminium alloys, Scripta Materialia 64 (2011) 916-918.

[32] V.C. Kannan, G. Thomas. Dislocation Climb and Determination of Stacking-Fault Energies in Al and Al-1\% Mg, Journal of Applied Physics 37 (1966) 2363-2370.

[33] R.R. Keller, R.H. Geiss. Transmission EBSD from $10 \mathrm{~nm}$ domains in a scanning electron microscope, Journal of Microscopy 245 (2012) 245-251.

[34] V. Randle. A methodology for grain boundary plane assessment by single-section trace analysis, Scripta Materialia 44 (2001) 2789-2794.

[35] S.I. Wright, R.J. Larsen. Extracting twins from orientation imaging microscopy scan data, Journal of Microscopy 205 (2002) 245-252.

[36] R.C. Pond, V. Vitek. Periodic Grain Boundary Structures in Aluminium. I. A Combined Experimental and Theoretical Investigation of Coincidence Grain Boundary Structure in Aluminium, Proceedings of the Royal Society of London A: Mathematical, Physical and Engineering Sciences 357 (1977) 453-470.

[37] V. Randle. The Measurement of Grain Boundary Geometry, Taylor \& Francis, 1993.

[38] X. Zhang, O. Anderoglu, A. Misra, H. Wang. Influence of deposition rate on the formation of growth twins in sputter-deposited 330 austenitic stainless steel films, Applied Physics Letters 90 (2007) 153101.

[39] X. Zhang, H. Wang, X.H. Chen, L. Lu, K. Lu, R.G. Hoagland, A. Misra. High-strength sputterdeposited Cu foils with preferred orientation of nanoscale growth twins, Applied Physics Letters 88 (2006) 173116.

[40] M. Ohring. Chapter 9 - Film structure. in: Ohring M, (Ed.). Materials Science of Thin Films (Second Edition). Academic Press, San Diego, 2002. pp. 495-558.

[41] K.S.S. Harsha. Principles of Vapor Deposition of Thin Films, Elsevier Science, 2005.

[42] C.V. Thompson. Grain Growth in Thin Films, Annual Review of Materials Science 20 (1990) 245-

268. 

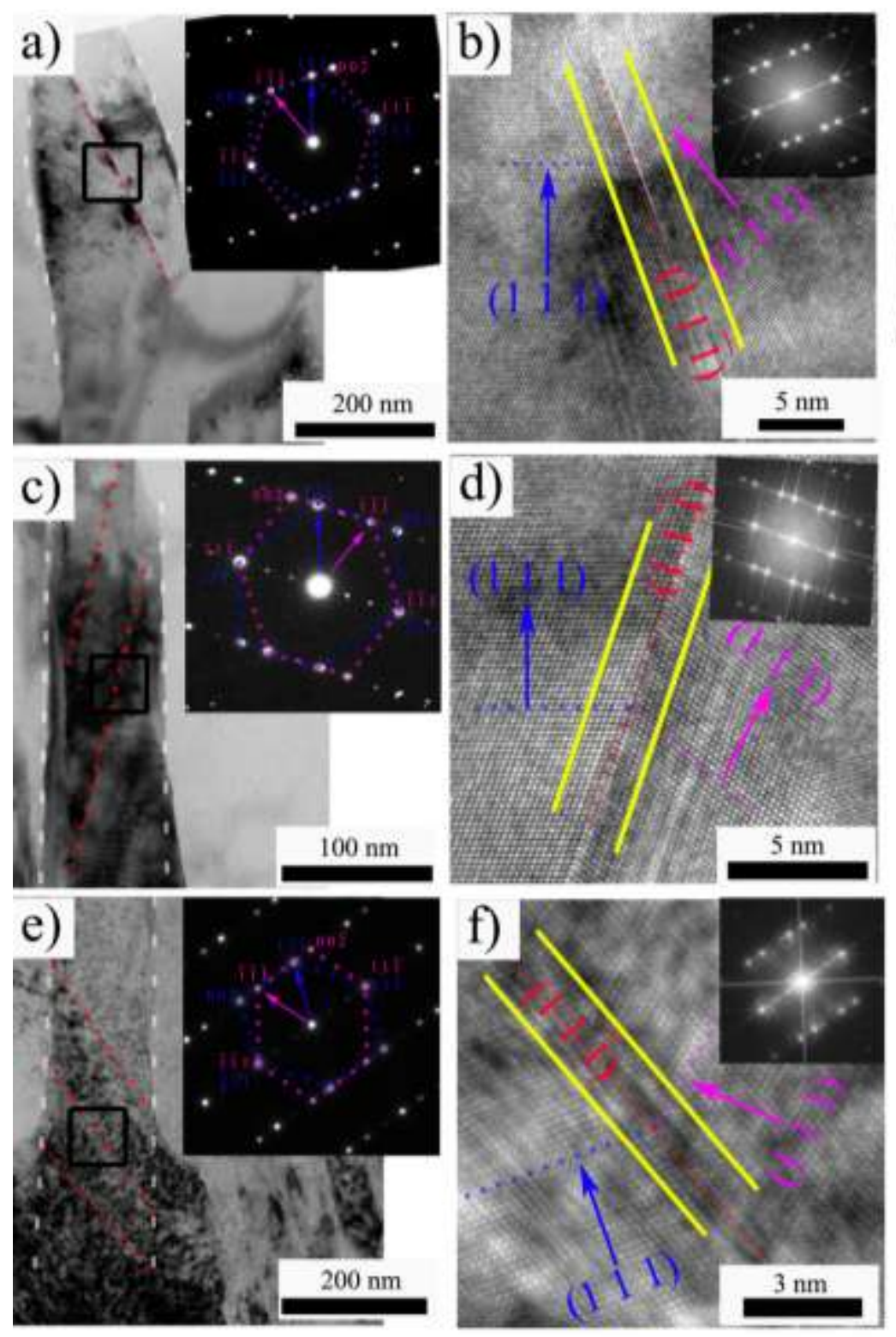

Figure 1. Low magnification and high resolution images of: $\mathrm{Al}$ (a and b), $\mathrm{Al}-\mathrm{Mg}$ (c and d), $\mathrm{Ni}$ (e and f). The columnar grains are marked by white dashed lines, while the red dotted lines mark an inclined TB (a, c, and e). The inset (a, c, and e) SAED patterns show the typical double hexagon formed due to the presence of TBs, the SAED patterns are decorated with blue and magenta dotted lines to form the double hexagon as a consequence of a reversal in the stacking sequence of the material, the diffraction spots are indexed according to the plane orientation. High 
resolution images of the square regions in a), c) and d) are presented in b), d) and e) respectively. The CTBs marked with red dotted lines lie in a $\left(\begin{array}{lll}1 & 1 & -1\end{array}\right)$ or $\left(\begin{array}{lll}-1 & -1 & 1\end{array}\right)$ plane, while the blue and magenta dotted lines show the $\left(\begin{array}{lll}1 & 1 & 1\end{array}\right)$ planes at each side of the two sides of the CTB. The insets in b), d) and f) are fast Fourier transforms of the high resolution image, notice the double hexagon typical of a CTB.
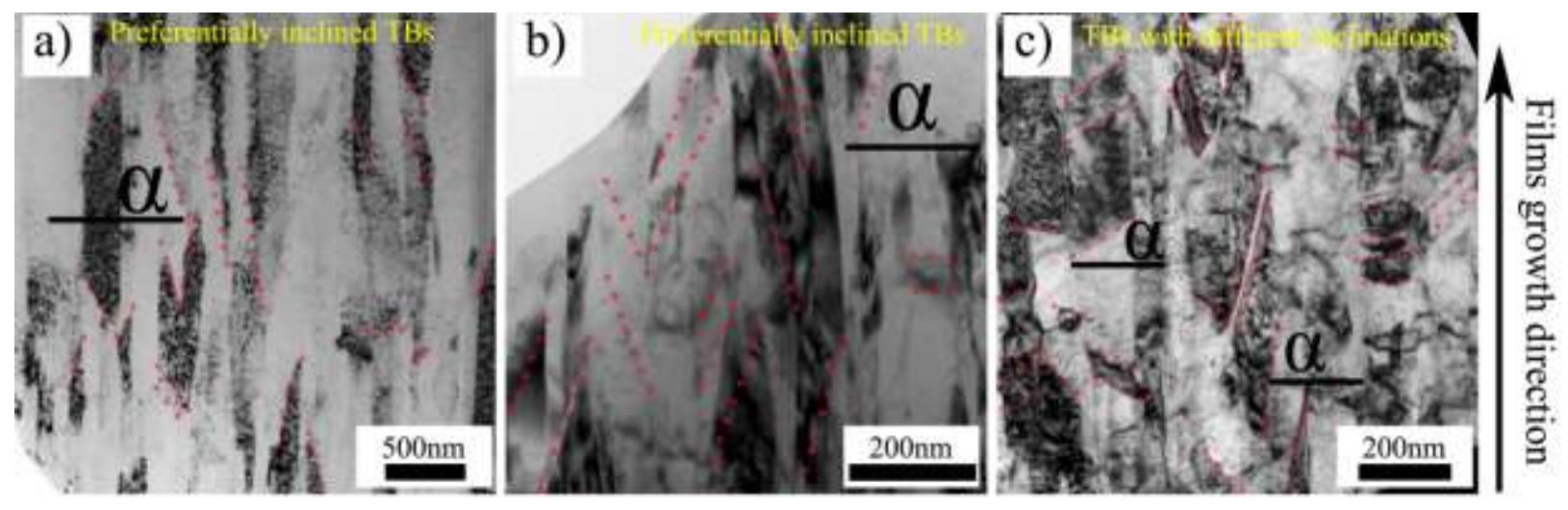

Figure 2. Representative cross-sectional TEM images of columnar grains with TBs of: a) Al, b) $\mathrm{Al}-\mathrm{Mg}$, and c) Ni. TBs are marked by the red dotted lines. $\mathrm{Al}$ and $\mathrm{Al}-\mathrm{Mg}$ have a preferential inclined TB angle $\alpha \sim 70^{\circ}$, in $\mathrm{Ni}$ the inclination of the TB angle $\alpha$ varies from $0^{\circ}$ to $\sim 75^{\circ}$. 


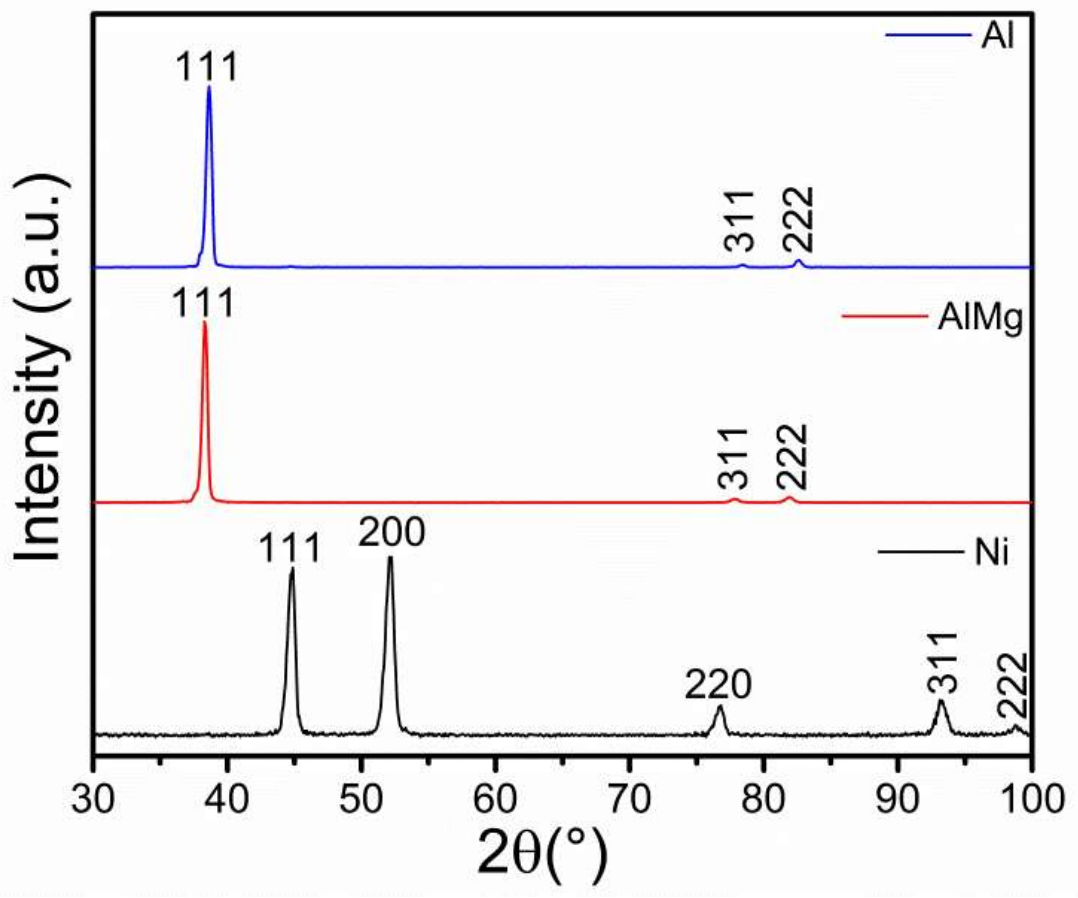

Figure 3. Normalized XRD patterns of the $\mathrm{Al}, \mathrm{Al}-\mathrm{Mg}$, and $\mathrm{Ni}$ films. $\mathrm{Al}$ and $\mathrm{Al}-\mathrm{Mg}$ have a strong $\{111\}$ texture, while Ni presents two strong textures. 


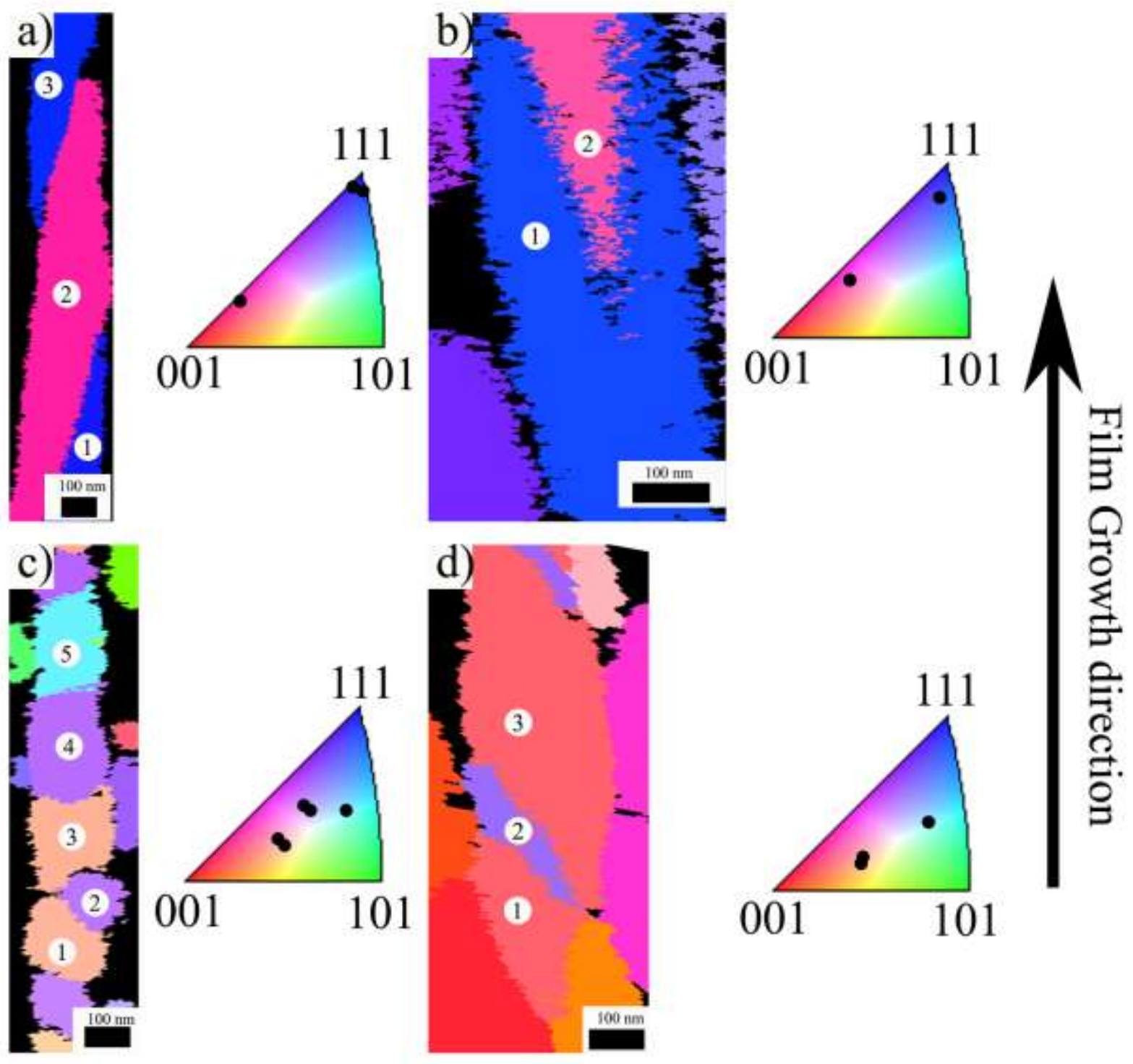

Figure 4. Cross-sectional T-EBSD scans of single columnar grains. a) Al, b) Al-Mg), c) and d) Ni. Notice that in $\mathrm{Al}$ and $\mathrm{Al}-\mathrm{Mg}$ the change in texture occurred between $\{111\}$ and $\{115\}$ planes, while in Ni the change in texture is from multiple plane orientations. Two examples are given for the Ni film to show horizontal and inclined TBs. (Colors correspond to the orientation of the grains in the film growth direction). 
Table 1. T-EBSD analysis of the CTBs in Al, Al-Mg, and Ni films.

\begin{tabular}{|c|c|c|c|c|c|c|}
\hline $\begin{array}{l}\text { Sample } \\
\text { name }\end{array}$ & $\begin{array}{c}\text { Figure } 4 \\
\text { label }\end{array}$ & $\begin{array}{l}\text { CTB between } \\
\text { the white } \\
\text { points in } \\
\text { Figure } 4\end{array}$ & $\begin{array}{c}\text { Plane before } \\
\text { CTB }\end{array}$ & $\begin{array}{l}\text { Plane after } \\
\text { CTB }\end{array}$ & $\begin{array}{l}\text { Missorientation } \\
\text { angle }\end{array}$ & $\begin{array}{c}\text { Plane } \\
\text { perpendicular to } \\
\text { Missorientation } \\
\text { axis } \\
\end{array}$ \\
\hline \multirow{2}{*}{$\mathrm{Al}$} & \multirow{2}{*}{ a) } & 1 and 2 & $\left(\begin{array}{lll}-1 & 1 & 1\end{array}\right)$ & $\left(\begin{array}{lll}-1 & 1 & 5\end{array}\right)$ & 59.8 & $\left(\begin{array}{lll}1 & -1 & 1\end{array}\right)$ \\
\hline & & 2 and 3 & $\left(\begin{array}{lll}-1 & 1 & 5\end{array}\right)$ & $\left(\begin{array}{lll}-1 & 1 & 1\end{array}\right)$ & 59.1 & $\left(\begin{array}{l}17-17 \\
-16\end{array}\right)$ \\
\hline $\mathrm{Al}-\mathrm{Mg}$ & b) & 1 and 2 & $\left(\begin{array}{lll}9 & -8 & 9\end{array}\right)$ & $(5-7$ 26) & 59.9 & $(1-1-1)$ \\
\hline \multirow{4}{*}{$\mathrm{Ni}$} & \multirow{4}{*}{ c) } & 1 and 2 & $\left(\begin{array}{lll}-3 & -1 & 8\end{array}\right)$ & $(-7-4-3)$ & 59.3 & $\left(\begin{array}{lll}1 & -1 & 1\end{array}\right)$ \\
\hline & & 2 and 3 & $(-7-4-3)$ & $\left(\begin{array}{lll}-5 & -2 & 13\end{array}\right)$ & 59.8 & $\left(\begin{array}{ll}17 & -18 \\
17\end{array}\right)$ \\
\hline & & 3 and 4 & $\left(\begin{array}{lll}-5 & -2 & 13\end{array}\right)$ & $(-9-5-4)$ & 59.4 & $\left(\begin{array}{lll}1 & -1 & 1\end{array}\right)$ \\
\hline & & 4 and 5 & $(-9-5-4)$ & (10 4 9) & 59.5 & $\left(\begin{array}{lll}1 & 1 & 1\end{array}\right)$ \\
\hline \multirow{2}{*}{$\mathrm{Ni}$} & \multirow{2}{*}{ d) } & 1 and 2 & $\left(\begin{array}{lll}4 & -2 & 21\end{array}\right)$ & $\left(\begin{array}{lll}-7 & 10 & -15\end{array}\right)$ & 60.0 & $\left(\begin{array}{lll}1 & 1 & -1\end{array}\right)$ \\
\hline & & 2 and 3 & $\left(\begin{array}{lll}-7 & 10 & -15\end{array}\right)$ & $(4-221)$ & 60.0 & $\left(\begin{array}{lll}1 & 1 & -1\end{array}\right)$ \\
\hline
\end{tabular}

Table 2. Microstructural properties of the Al, Al-Mg and Ni films.

\begin{tabular}{cccccc}
$\begin{array}{c}\text { Sample } \\
\text { name }\end{array}$ & $\begin{array}{c}\mathrm{SFE} \\
\left(\mathrm{mJ} / \mathrm{m}^{2}\right)\end{array}$ & $\begin{array}{c}\text { Density of } \\
\text { TBs per unit } \\
\text { area } \\
\left(\mathrm{TBs} / \mu \mathrm{m}^{2}\right)\end{array}$ & $\begin{array}{c}\text { Total area } \\
\text { analyzed } \\
\left(\mu \mathrm{m}^{2}\right)\end{array}$ & $\begin{array}{c}\text { Fraction of } \\
\text { twinned grains } \\
(\%)\end{array}$ & $\begin{array}{c}\text { Average grain } \\
\text { width } \\
(\mathrm{nm})\end{array}$ \\
\hline $\mathrm{Al}$ & 166 & 3.4 & 151 & 46 & $290 \pm 40$ \\
$\mathrm{Al}-\mathrm{Mg}$ & $<166$ & 47 & 11 & 70 & $90 \pm 10$ \\
$\mathrm{Ni}$ & 125 & 69 & 20.7 & 90 & $70 \pm 10$ \\
\hline
\end{tabular}

